\title{
Neonatal bilateral diaphragmatic paralysis caused by brain stem haemorrhage
}

\author{
S BLAZER, ${ }^{*}$ J A HEMLI, $\dagger$ P O SUJOV,${ }^{*}$ AND J BRAUN $\ddagger$
}

${ }^{*}$ Division of Neonatal Medicine, Department of Pediatrics, + Pediatric Neurology Unit, and $\ddagger$ Department of Diagnostic Radiology, Rambam Medical Center, and Faculty of Medicine, Technion - Israel Institute of Technology, Haifa, Israel

SUMmaRY We describe a neonate with severe bilateral diaphragmatic paralysis caused by haemorrhage in the lower brain stem. To our knowledge this association has not been previously reported in the English medical literature.

Bilateral diaphragmatic paralysis in the neonate is a life threatening situation. ${ }^{12}$ It is usually a result of birth trauma to the cervical nerve roots or cervical spine, although agenesis of the phrenic nerve has also been reported, ${ }^{1}$ and in some cases no cause could be determined. ${ }^{23}$

\section{Case report}

A boy, weighing $3600 \mathrm{~g}$, was delivered at 42 weeks' gestation after an uncomplicated pregnancy. After delivery intubation was required because of delayed onset breathing, and the endotracheal tube was suctioned because of the presence of heavy meconium stained amniotic fluid. The Apgar scores were 4 and 8 at 1 and 5 minutes, respectively. Physical examination after delivery showed mild respiratory distress, and there was mild hypotonia of both legs. No signs of brachial plexus damage were noted.

At 1 hour of life respiratory distress increased and paradoxical respiratory movements were noted. Values for blood gases were within the normal range. A chest radiograph showed elevation of both diaphragms to the level of the fifth rib, without signs of meconium aspiration (fig 1). Chest fluoroscopy showed no spontaneous diaphragmatic movements, but there was paradoxical upward movement of both diaphragms during inspiration. Respiratory difficulty increased, and mechanical ventilation had to be instituted.

At 14 hours of life the sucking reflex disappeared. Ultrasonography showed no intracranial haemorrhage; the spinal fluid was normal. Computed tomography of the head, performed on the 10th day of life (fig 2) showed a large asymmetric area of increased density in the medulla oblongata extending more to the right, a finding that was interpreted

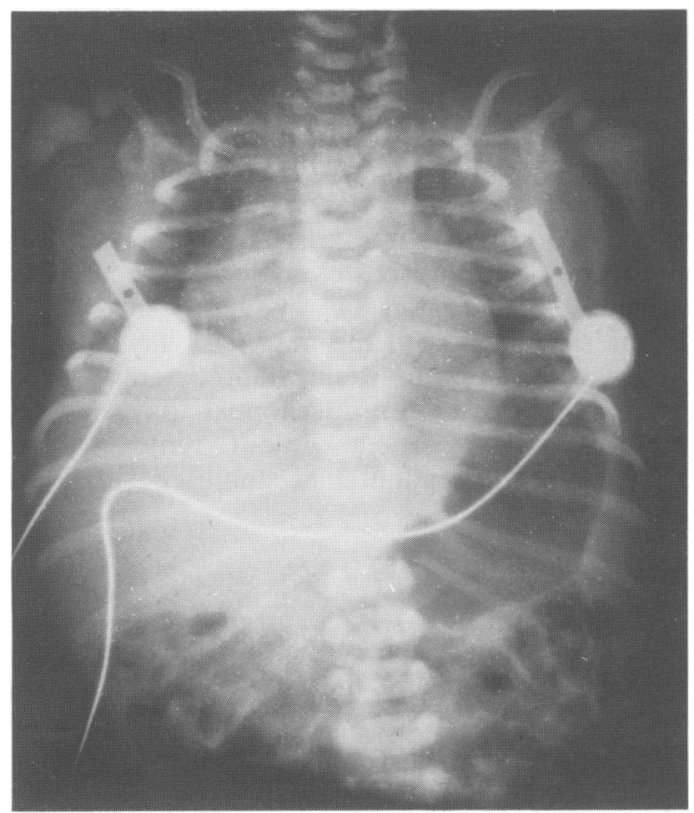

Fig 1 Radiograph of chest on the 1st day of life showing the high position of both diaphragms.

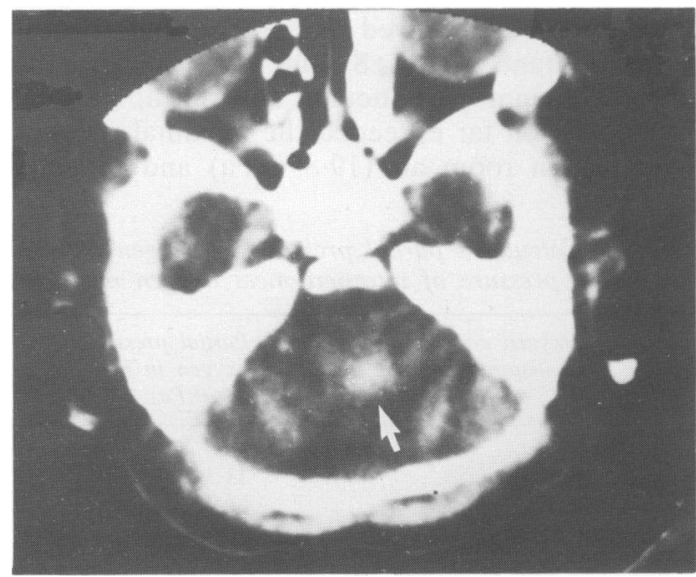

Fig 2 First computed tomogram showing hyperdense region within the right medulla oblongata (arrow). 
as haemorrhage. The pons, fourth ventricle, and basal cisterns were normal.

On the 3rd week of life, generalised hypotonia was observed and there were fasciculations of the tongue. Electromyographic studies showed normal conduction velocities in the extremities but there were signs of denervation (fibrillation potentials) in the sternocleidomastoid and trapezius muscles. Attempts to evoke phrenic nerve activity failed. Muscle enzymes values were within normal limits. Histological and electron microscopical studies of muscle gave normal results.

At the age of 6 weeks, the fasciculations of the tongue subsided. A repeat electromyographic study showed diaphragmatic activity after stimulation of the phrenic nerve, but there were still no spontaneous diaphragmatic movements. A computed tomogram of the head showed resorption of the haemorrhagic focus in the medulla, with a reduction in brain stem size and widening of prepontine and quadrigeminal cisterns. Higher sections showed diffuse atrophic changes. At the age of 7 months the computed tomogram showed more pronounced cerebral atrophic changes.

At 3 months of life the baby could be weaned from the respirator, although moderate respiratory difficulty persisted over the next several months. Sucking and swallowing ability improved steadily.

Partial diaphragmatic paralysis persisted until the age of 11 months, when breathing became practically normal, with return of the diaphragms to a normal position on chest radiography and fluoroscopy. During the above period, the infant received intensive respiratory physiotherapy and stimulation. His mental development progressed satisfactorily, but gross motor achievements remained slightly delayed.

\section{Discussion}

The diaphragm has an essential role as a respiratory muscle in neonates. The major changes in thoracic volume in neonates are the result of diaphragmatic contraction. ${ }^{1}$ Diaphragmatic malfunction in neonates, by either a primary diaphragmatic muscular problem or secondary to phrenic nerve paralysis (unilateral or bilateral), may produce severe cardiopulmonary embarrassment and may result in death if early supportive treatment is not carried out. ${ }^{1}$ Brain stem haemorrhage has not previously been reported as a cause of paralysis of the diaphragm in the neonate.

Posterior fossa haemorrhages are rare in the newborn. In one series 17 neonates out of 700 were found to have a posterior fossa haemorrhage on computed tomography (six of these infants were premature $).{ }^{4}$ These posterior fossa haemorrhages were either in the subdural space or intracerebellar. Brain stem haemorrhage was not present in any of them. The prognosis in these neonates was poor: seven died, and only three of the survivors were normal at follow up. In another series, in a postmortem study of 105 neonates and fetuses, intracerebellar haemorrhage was found in two cases; the pathogenesis of the posterior fossa haemorrhage was attributed to birth trauma or prematurity. ${ }^{5}$ The aetiology of posterior fossa haemorrhage in the premature may be similar to that of the subependymal haemorrhage in the cerebral hemispheres. ${ }^{4}$ Perinatal asphyxia and hypoxic-ischaemic brain damage may be an important cause of posterior fossa haemorrhage, ${ }^{6}$ and may also explain the development of cerebral atrophy in our case. In the English medical literature we could not find another case in which the diagnosis of brain stem haemorrhage was made during life.

In the present case, the baby suffered from perinatal distress with respiratory difficulty immediately after birth, and bilateral diaphragmatic paralysis was diagnosed. Cervical trauma causing phrenic nerve injury could be excluded; peripheral phrenic injury was unlikely, as cervical phrenic nerve stimulation resulted in diaphragmatic contractions. The computed tomogram disclosed a large haemorrhage within the medulla oblongata; an insult to this strategic neurological site may cause severe functional or structural damage endangering the newborn's life. The motor cranial nuclei IX, X, XI, and XII in the medulla oblongata programme and activate the phrenic and intercostal nerves. The motor neurons to the phrenic nerve (in $\mathrm{C} 3, \mathrm{C} 4$, and C5), to the intercostal muscles and presumably, to the accessory muscles of respiration are all under the control of respiratory centres in the medulla oblongata. These may have become damaged by the haemorrhagic lesion in our patient.

This infant displayed respiratory distress due to neurogenic diaphragmatic paralysis, which improved and returned to normal over the following months, after resorption of a medullary haemorrhage. The temporary clinical appearance of fasciculations of the tongue and electrophysiological fibrillations in the muscles innervated by the accessory nerve are fully consistent with the localisation of the haemorrhage in the medulla, with functional injury of the IX, X, and XIII motor nuclei.

The commonly recognised causes of diaphragmatic paralysis are local pathology of the diaphragm (muscular hypoplasia) or phrenic nerve pathology (injury or agenesis). This report describes a higher level, intracranial pathology, as a cause of peripheral functional diaphragmatic paralysis. The 
experience with our case suggests that careful neurologic assessment, including computed tomography or magnetic resonance imaging, is indicated in the patient with diaphragmatic paralysis of unknown origin.

\section{References}

${ }^{1}$ Aldrich TK, Herman JH, Rochester DF. Bilateral diaphragmatic paralysis in the newborn infant. J Pediatr 1980;97:988-91.

2 Wexler HA, Poole CA. Neonatal diaphragmatic dysfunction. AJR 1976;127:617-22.

${ }^{3}$ Haller JA, Pickard LR, Tepas JJ, et al. Management of diaphragmatic paralysis in infants with special emphasis on selection of patients for operative plication. J Pediatr Surg 1979;14:779-84.

4 Scotti G, Flodmark O, Harwood-Nash DC, Humphries RP. Posterior fossa hemorrhages in the newborn. J Comput Assist Tomogr 1981;5:68-72.

5 Ludwig B, Becker K, Rutter G, Bohl Y, Brand M. Postmortem $\mathrm{CT}$ and autopsy in perinatal intracranial hemorrhage. AJNR 1983;4:27-36.

${ }^{6}$ Lon HC. Perinatal hypoxic-ischemic brain damage and intraventricular hemorrhage; a pathogenetic model. Arch Neurol $1980 ; 37: 585-7$.

Correspondence and requests for reprints to Dr S Blazer, Division of Neonatology, Rambam Medical Center, Haifa 35254, Israel.

Accepted 18 July 1987 